\title{
USEFULNESS OF GOLD NANOPARTICLES AS LABELS FOR THE DETERMINATION OF GLIADINS BY IMMUNOAFFINITY CHROMATOGRAPHY WITH LIGHT SCATTERING DETECTION
}

\author{
M.A. Molina-Delgado, M.P. Aguilar-Caballos, A. Gómez-Hens \\ Department of Analytical Chemistry \\ University of Córdoba \\ Campus of Rabanales \\ Annex to Marie Curie building \\ 14071-Córdoba \\ Spain \\ E-mail:qa1gohea@uco.es \\ Web: http://www.uco.es/FQM-303/
}

\begin{abstract}
A simple and fast immunoaffinity method is proposed for the determination of gliadins for the first time using gold nanoparticles (AuNPs) as labels. The tracer used consists in a gliadin-gold nanoparticle conjugate prepared by the adsorption of gliadins onto the nanoparticle surface. This approach prevents the use of chemical reactions for the covalent linking of the protein to the nanoparticle, which minimizes potential denaturation processes during the coupling process. The proposed method relies on the injection in a commercial Protein G column of a preincubated mixture containing gliadins, polyclonal anti-gliadin antibodies, and the gliadin-gold nanoparticle tracer.
\end{abstract}

Two different tracers, using AuNPs with diameters of 10-nm and 20-nm, were synthesized in order to compare their features for the development of the method. This approach allows the separation of free and bound tracer fractions without any additional elution step, and the direct measurement of the light scattering intensity of the free tracer through the peak height of the immunochromatogram, which is proportional to the analyte concentration. The immunocolumn can be used up to 25 times without eluting and regenerated for at least 20 times. The dynamic ranges of the method are 0.5-15.0 and 1.5 - 15.0 $\mu \mathrm{g}$ $\mathrm{mL}^{-1}$ gliadins, using 20-nm and 10-nm Au-NPs as labels, respectively. The detection limits, calculated according to IUPAC recommendations were $0.2 \mu \mathrm{g} \mathrm{mL}^{-1}$ and $0.8 \mu \mathrm{g} \mathrm{mL}^{-1}$ gliadins, for $20-\mathrm{nm}$ and $10-\mathrm{nm}$ AuNP-gliadin tracer, respectively. The precision, expressed as relative standard deviation, ranges between 2.7 and $2.9 \%$ using 20-nm AuNPs and 4 and $6.1 \%$ for $10-n m$ AuNPs. The method has been applied to the analysis of beer samples by treating them with ethanol for the removal of alcohol insoluble compounds from sample matrix which, unlike gliadins, are not soluble in this medium.

This study contributes to expand the versatility of AuNPs in bioassays and, more specifically, to the applications of protein-AuNPs interactions. A very simple immunoaffinity procedure with a relatively high sample throughput has been achieved by using commercial pre-packed Protein G columns and preincubated mixtures of the immunoreagents and samples. The multiple incubation and washing steps that are usually needed for the performance of competitive and sandwich ELISA methods used for the determination of gliadins, are avoided using this method. 